\title{
Flutuação Populacional de Rhynchophorus palmarum L. (Coleoptera: Curculionidae) em Plantios de Palma de Óleo em Roraima
}

\author{
Ruy Guilherme Correia ${ }^{\bowtie}$, Antonio Cesar Silva Lima², Antonio Carlos Centeno Cordeiro ${ }^{3}$, \\ Francisco Clemilto da Silva Maciel ${ }^{4}$, Walmer Bruno Rocha Martins ${ }^{1}$ \& Luiz Fernandes Silva Dionísioº
}

1. Universidade Federal Rural da Amazônia, e-mail: cciigg25@yahoo.com.br (Autor para correspondência ${ }^{\bowtie}$ ), walmerbruno@yahoo. com.br. 2. Universidade Federal de Roraima, e-mail: ant.cesar@uol.com.br, fernandesluizo3@gmail.com. 3. Embrapa Roraima, e-mail: acarlos@cpafrr.embrapa.br. 4. Empresa Palmaplan Agroindustrial LTDA, e-mail: francisco.dende@hotmail.com.

\section{EntomoBrasilis 8 (2): 130-134 (2015)}

Resumo. Esta pesquisa teve como objetivo avaliar a flutuação populacional de Rhynchophorus palmarum L. (Coleoptera: Curculionidae) em plantios de Elaeis guineensis Jacq, em ecossistemas de savana e floresta no estado de Roraima. A pesquisa foi desenvolvida durante o período de janeiro de 2011 a fevereiro de 2012 nos campos experimentais da Embrapa Roraima: Monte Cristo e Caroebe, numa área de 2 hectares com a cultura da Palma de Óleo, em cada campo. Foram distribuídas armadilhas iscadas com roletes de cana-de-açúcar e feromônio de agregação para captura dos insetos no entorno dos plantios. Ocorreu flutuação de $R$. palmarum com picos populacionais nos meses de julho e agosto no ecossistema de savana e de abril a setembro no ambiente de floresta. A população de $R$. palmarum foi menor em meses de baixa precipitação pluviométrica nos dois ambientes avaliados com a Palma de Óleo em Roraima

Palavras-Chave: Broca-do-coqueiro; Dinâmica populacional; Feromônio; Monitoramento.

Population Fluctuation of Rhynchophorus palmarum L. (Coleoptera: Curculionidae) in Plantations of Oil Palm in Roraima

Abstract. This research aimed to evaluate the population fluctuation Rhynchophorus palmarum L. (Coleoptera: Curculionidae) in Elaeis guineensis Jacq plantations in forest and savanna ecosystems in the state of Roraima. The research was conducted during the period from January 2011 to February 2012 in the experimental field of Embrapa Roraima: Monte Cristo and Caroebe in an area of 2 hectares with culture Oil Palm in each field. Traps baited with rollers cane sugar and aggregation pheromone for capturing insects in the vicinity of the plantations were distributed. Fluctuation occurred R. palmarum with population peaks in the months of July and August in the savanna ecosystem and from April to September in the forest environment. The population of $R$. palmarum was lower in months of low rainfall in the two environments assessed with a palm-oil-in Roraima.

Keywords: Borer coconut; Monitoring; Pheromone; Population dynamics.

Palma de Óleo (Elaeais guineensis Jaqc) é uma palmeira de origem africana cultivada em regiões tropicais úmidas na África, Ásia e América, e representa a segunda mais importante fonte de óleo vegetal (SANTOS 2010). No entanto, os problemas fitossanitários são um dos fatores limitantes na exploração da palmicultura, por serem de relevante expressão econômica (DUARTE et al. 2008).

Entre as pragas que causam prejuízos econômicos para a Palma de Óleo em todo o Brasil o Coleoptera Rhynchophorus palmarum L. destaca-se como a principal espécie de inseto que prejudica esta cultura. Este curculionidae provoca danos diretos, causados pelas larvas que se alimentam de estruturas da planta, e indiretos, por serem vetores do nematóide Bursaphelenchus cocophilus (Cobb) causador da doença do anel-vermelho (KRUG et al. 2013). Este nematóide pode causar perda anual de até $15 \%$ de um plantio de E. guineensis (CHINCHILLA 1992).

O coleóptero $R$. palmarum é considerado importante praga da cultura E. guineensis desde o século XVII, onde ficou conhecida popularmente como bicudo-do-coqueiro (FERREIRA et al. 2003). Os adultos apresentam longevidade de 45 a 60 dias e as fêmeas ovipositam uma média de 100 a 400 ovos durante o ciclo de vida, a oviposição é feita na região da coroa e nas partes tenras das bases das folhas no estipe da planta (Moura et al. 2006).

Os danos causados pelos R. palmarum ocorrem principalmente nas plantas que apresentam alguma deficiência física ou fisiológica, devido a fermentação de sua seiva exalar o odor que atrai os besouros da mesma espécie (GALlo et al. 2002). Já em Bactris gasipaes Kunth (Arecales: Arecaceae) o ataque acontece preferencialmente no estipe, quando é efetuado o corte para colheita do palmito (ZoRZENON et al. 2000).

O controle do R. palmarum é normalmente realizado por meio da aplicação de inseticidas nos ferimentos das plantas ou utilizando fungos entomopatogênicos e insetos parasitas (FERREIRA et al. 2003; Moura et al. 2006). O controle cultural ocorre pela eliminação de plantas com sintomas do anel-vermelho, enquanto que o controle comportamental é feito através de feromônios de agregação específico para a espécie (OEHLSCHLAGER et al. 2002).

O controle comportamental apresenta melhor resultado na captura dos insetos quando é feito através de armadilhas com o uso de feromônio de agregação mais o atrativo alimentar como isca atraente na captura de $R$. palmarum, sendo recomendada a combinação cana-de-açúcar mais feromônio de agregação, em armadilhas tipo balde (NAVARRo et al. 2002 \& DUARTE et al. 2003).

É muito importante para o sucesso do controle de pragas o conhecimento da flutuação populacional das mesmas no ambiente, segundo CosTA et al. (2014) a flutuação populacional é o estudo das variações na distribuição e abundância de espécies 


\section{e floresta).}

Conforme Silveira Neto et al. (1995), é praticamente improvável saber o número total de insetos existente em um ambiente, e o monitoramento destes indivíduos podem então ser realizado mediante a estimativa da população por meio de amostragem, que é uma das etapas fundamentais em estudos de ecologia quantitativa. O conhecimento da flutuação populacional de uma praga passa a ser uma alternativa para o melhor controle e para a diminuição de impactos ambiental causado pelo excessivo uso de defensivos agrícolas (FACHINELLo et al. 2003).

De acordo com SANwaYs (1995), o levantamento e a flutuação populacional de insetos são importantes para tomadas de decisão em caso de surtos de pragas. O monitoramento desses indivíduos pode facilitar a escolha do melhor controle na tomada de decisão, além de se obter informações sobre a diversidade da entomofauna benéfica de um ambiente (Thomazinı \& Thomazin 2000).

O monitoramento populacional do inseto $R$. palmarum torna possível a escolha do tipo de manejo a ser aplicado e a eficiência do mesmo, sendo fundamental no conhecimento do comportamento desta espécie no ambiente, o qual é capaz de diminuir o número de insetos em plantios de E. guineensis reduzindo as ocorrências da doença do anel-vermelho nessas áreas (OEHLSCHLAger et al. 2002). Neste sentido, objetivou-se nesta pesquisa avaliar a flutuação populacional do inseto $R$. palmarum em plantios de Palma de Óleo em ecossistemas de savana e floresta no estado de Roraima.

\section{MATERIAL E MÉTODOS}

O experimento foi realizado em plantios de Palma de Óleo no período de fevereiro de 2011 a janeiro de 2012 em ambiente de savana (cerrado) e floresta no estado de Roraima. O plantio do ambiente de savana está localizado no Campo Experimental Monte Cristo pertencente à Embrapa-Roraima (60 ${ }^{\circ} 42^{\prime} 40^{\prime \prime} \mathrm{W}$ e $02^{\circ} 56^{\prime} 53$ ” N), município de Boa Vista, com uma área de 2,08 ha (população de 143 plantas/ha-1, espaçamento de $9 \mathrm{~m} \times 9 \mathrm{~m}$, em arranjo de triangulo equilátero), instalada em maio de 2007, o local do experimento apresenta a fitofisionomia do tipo savana gramíneo-lenhosa.

O clima é do tipo Awi de acordo com a classificação de Köppen, tropical chuvoso, com duas estações bem definidas, sendo uma chuvosa (abril a agosto) e a outra com menor período de ocorrência de chuvas (outubro a março) e temperatura, precipitação e umidade relativa do ar com média anual de 27,4 ${ }^{\circ} \mathrm{C}, 1.678 \mathrm{~mm}$ e $70 \%$, respectivamente (ARAúso et al. 2001).

O plantio do ambiente de floresta está localizado na Fazenda Califórnia ( $59^{\circ} 42^{\prime} 33,5$ ” W e $00^{\circ} 45^{\prime} 50,1$ ” N), no município de Caroebe a $350 \mathrm{~km}$ de Boa Vista, com uma área de 2 ha (população de 143 plantas $/ \mathrm{ha}^{-1}$, espaçamento de $9 \mathrm{~m}$ x $9 \mathrm{~m}$, em arranjo de triangulo equilátero), instalados em junho de 2006. O bioma deste local é o Amazônico, apresentando o clima do tipo Ami de acordo com a classificação de Köppen, ou seja, tropical chuvoso, com temperatura média entre 25 e $28^{\circ} \mathrm{C}$ e precipitação pluviométrica anual variando de 1.800 a $1.900 \mathrm{~mm}$ e umidade relativa do ar entre 85 a $90 \%$ (BARBOSA 1997).

Para o experimento foram utilizadas armadilhas compostas por baldes plásticos de 30 L fechado com uma tampa que apresentava duas aberturas de $10 \mathrm{~cm}$ de diâmetro, acopladas ao funil plástico com o estreitamento final de $1,5 \mathrm{~cm}$ de diâmetro segundo metodologia de (Chinchilla \& OHLSChlager 1992). O fundo das armadilhas apresentavam orifícios de $2 \mathrm{~mm}$ de diâmetro que permitia o escoamento do excesso da água de chuva.

As armadilhas foram instaladas em volta do perímetro de cadaárea experimental, sendo 2 armadilhas/ha-1 espaçadas a intervalos de $300 \mathrm{~m}$, totalizando 4 armadilhas para cada ecossistema (savana
Em cada armadilha foram colocados como iscas atrativas, duas cápsula com o feromônio Rinchoforol (2 (E)-6-metil-2-hepta-4ol), trocadas a cada 60 dias, como recomendado pelo fabricante, além de 15 roletes de cana-de açúcar de $20 \mathrm{~cm}$ de comprimento que foram amassados e renovados a cada quinzena para facilitar a volatilização dos odores responsáveis pela atração do $R$. palmarum (Tiglia et al. 1998).

A intervalos de 15 dias, eram efetuadas as coletas e contagem dos adultos de R. palmarum atraídos e preso nos baldes, e a cada mês foi feito o somatório do número de espécimes capturados para a obtenção da flutuação populacional do inseto. Os besouros capturados foram alocados em recipientes plásticos e transportados para o Laboratório de Entomologia da Universidade Federal de Roraima.

Os dados meteorológicos diários, precipitação pluvial, temperatura média do ar e umidade relativa do ar, foram obtidos com a finalidade de estabelecer correlações entre os parâmetros climáticos e a flutuação populacional do besouro, estes dados climáticos foram oriundos de estações agrometeorológicas da Embrapa Roraima instaladas nos municípios de Caroebe e Boas Vista.

Para expressar a frequência mensal de $R$. palmarum capturado nas armadilhas foram considerados picos populacional quando ocorreu número $\geq$ a 70 insetos/armadilha/mês. Para verificar diferenças entre os ambientes de savana e floresta, os dados de densidade de $R$. palmarum e de clima foram submetidos a ao teste "t" de Student $(\mathrm{p}<0,05)$.

Para verificação a influência dos fatores climáticos sobre a abundância dos besouros foi feita a correlação de Pearson e análise de regressão múltipla com seleção de variáveis pelo método "stepwise" $(\mathrm{p}<\mathrm{0}, 05)$.

Foram utilizados os dados de média de temperatura e umidade relativa e o total de chuva acumulada do mês anterior às coletas dos insetos nas armadilhas. Os dados de precipitação pluvial e temperatura média do ar foram transformados em log $(x+1)$ e os de umidade relativa do ar transformados em arcoseno $(\mathrm{x}+1)^{1 / 2}$.

\section{RESULTADOS E DISCUSSÃO}

Houve diferença significativa na abundância de $R$. palmarum nos plantios de Palma de Óleo nos ambientes de savana e floresta para o período avaliado. Com relação aos fatores climáticos, verificaram-se diferenças significativas nos ecossistemas estudados, exceto para temperatura média do ar (Tabela 1).

A pluviosidade desenvolve grande importância na biologia de R. palmarum, em muitos casos sendo o fator abiótico que mais interfere no desenvolvimento populacional desta espécie (DUARTE et al. 2003). Neste experimento buscou-se correlacionar o total de insetos capturados nas armadilhas com cana-de-açúcar + feromônio de agregação, com os índices pluviométricos mensais de cada ambiente para se conhecer o nível de interferência da precipitação pluvial na flutuação populacional desta espécie nos ecossistemas de savana e floresta (Figuras 1 e 2).

Pela análise verificou-se que a população de $R$. palmarum apresentou oscilações durante o ano, havendo decréscimo populacional nos meses de baixo índice pluviométrico, assim como houve um aumento populacional dos besouros nos períodos em que os índices de chuvas foram maiores nas áreas experimentais (Figuras 1 e 2).

O comportamento populacional dos besouros verificado nos diferentes ecossistemas nesta pesquisa corrobora com os resultados de (Morales \& Chinchilla 1991; Ferreira et al. 2003) que em diferentes experimentos envolvendo o inseto $R$. 
Tabela 1. Média \pm Erro padrão de R. palmarum capturados por armadilhas em plantios de palma-de-óleo e variáveis climáticas (total de chuva e média da UR e temperatura média do ar), nos ecossistemas de savana e floresta em Roraima, de fevereiro de 2011 a janeiro de 2012.

\begin{tabular}{ccccc}
\hline Ecossistema & Rhynchophorus palmarum & Chuva $(\mathrm{mm})$ & UR (\%) & Temp. Média $\left({ }^{\circ} \mathrm{C}\right)$ \\
\hline Savana & $56,08 \pm 2,08 \mathrm{a}$ & $1520,90 \mathrm{~b}$ & $61,30 \mathrm{~b}$ & $28,70 \mathrm{a}$ \\
Floresta & $76,08 \pm 2,03 \mathrm{~b}$ & $2384,60 \mathrm{a}$ & $74,90 \mathrm{a}$ & $28,60 \mathrm{a}$ \\
\hline
\end{tabular}

Médias seguidas de mesma letra na coluna não diferem entre si pelo teste " $\mathrm{t}$ " de Student $(\mathrm{p}<0,05)$.

\section{Savana}

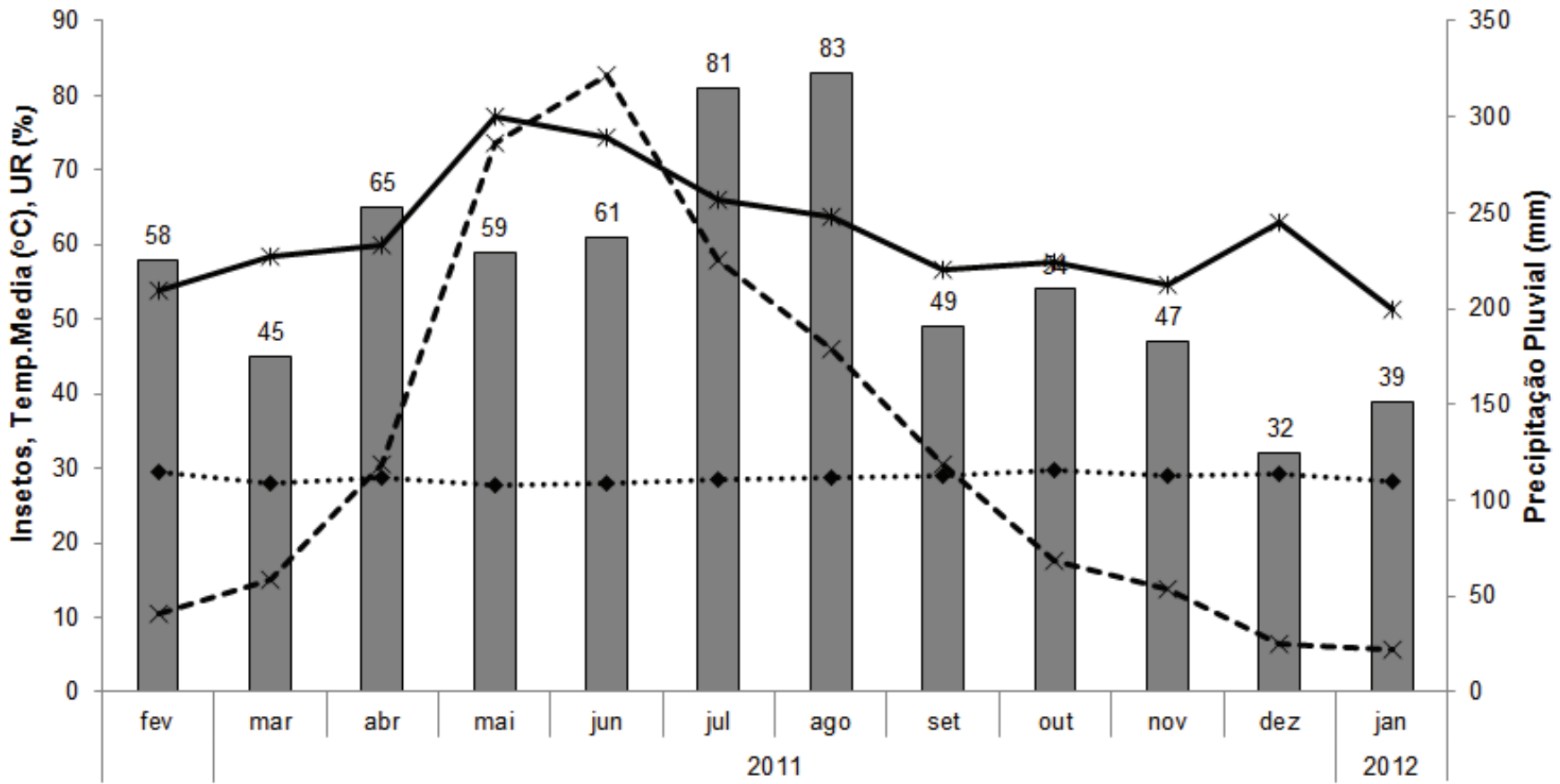

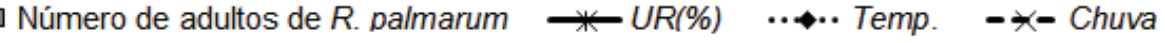

Figura 1. Flutuação populacional de adultos de Rhynchophorus palmarum em plantio de palma-de-óleo e dados climáticos no ambiente de savana (cerrado) no município de Boa Vista, Roraima, no período de fevereiro de 2011 a janeiro de 2012.

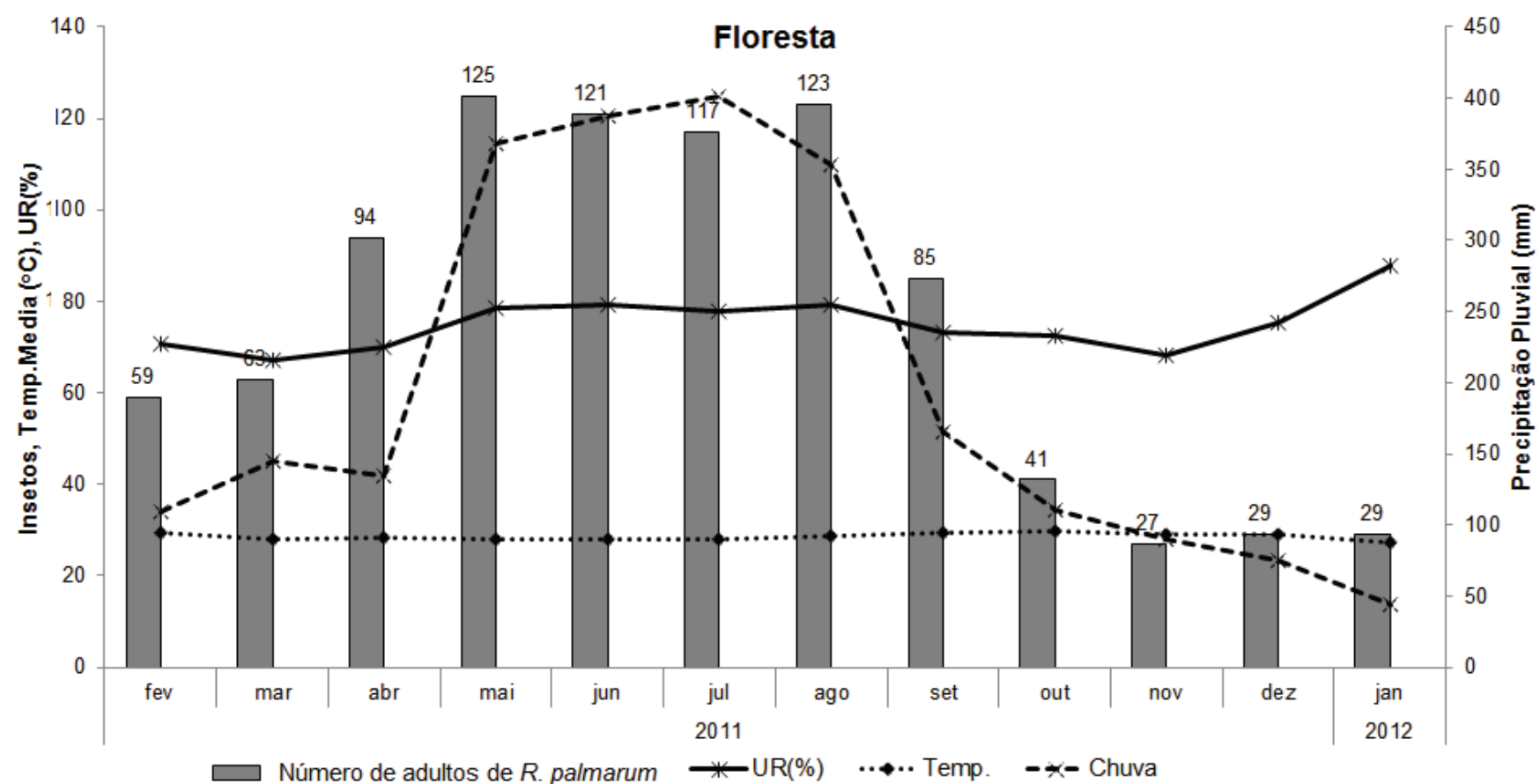

Figura 2. Flutuação populacional de adultos de Rhynchophorus palmarum em plantio de palma-de-óleo e dados climáticos no ambiente de floresta no município de Caroebe, Roraima, no período de fevereiro de 2011 a janeiro de 2012. 
T palmarum associado a várias espécies de plantas da família Arecaceae encontraram uma relação positiva entre a flutuação populacional do curculionídeo e o aumento da pluviosidade nas regiões estudadas. Esses autores observaram que o aumento da população de $R$. palmarum concentrava-se no período da estação chuvosa, atribuindo o fato de que, com altos índices de chuvas, as fibras vegetais das plantas que formam o casulo são facilmente abertas pelo inseto no momento de sua emergência.

Segundo TAKADA et al. (2011) em pesquisa com a referida praga em plantios com a cultura da banana em São Paulo, concluíram que a população de $R$. palmarum teve aumento na estação seca, fato que pode estar relacionado com o menor índice de chuvas na região, período que segundo os mesmos autores é favorável para a volatilização dos odores oriundos do atrativo alimentar e feromônio de agregação presente nas armadilhas. PINHo (2015) trabalhando com $R$. palmarum em agroecossistema de Palma de Óleo no estado do Pará conclui que a flutuação populacional do R. palmarum foi afetada pela variação de precipitação. Segundo o mesmo autor a população da praga tende a aumentar nos meses de maior estiagem.

Em estudos com flutuação populacional de $R$. palmarum em Palma de Óleo no estado do Amazonas Cysne et al. (2013) verificou que a quantidade de insetos não variou ao longo do ano de 2013, segundo os autores este efeito pode ser explicado pela pouca variação de temperatura e umidade caracterizando uma regularidade pluviométrica na região amazônica.

Para os fatores precipitação pluviométrica e o número de $R$. palmarum capturadosnas armadilhas, os resultadosapresentados neste trabalho não corroboram com BulgarelLi-Mora et al. (1998) que em duas áreas experimentais com a cultura da Palma de Óleo na Costa Rica realizaram avaliações usando armadilhas do tipo balde com o atrativo alimentar (cana-de-açúcar) mais o feromônio de agregação, e observaram uma correlação negativa entre a pluviosidade e o número de curculionídeos capturados durante um ano em ambas as áreas. Foram observados nesta pesquisa diferentes picos populacional de $R$. palmarum nas áreas de savana e floresta, podendo estes resultados terem sido afetado por diferentes fatores, como a maior diversidade de espécies de plantas tropicais, proximidade de mata natural, plantio de palmeiras próximas as áreas experimentais e fatores ambientais, entre outros.

Segundo AlpizAr et al. (2006), estudos de campo realizado em plantio de pupunha (B. gasipaes), na Costa Rica, indicaram que a ocorrência da espécie $R$. palmarum tende a diminuir no decorrer do tempo e que essa variação pode ser atribuída à retirada de um grande número de indivíduos devido a ação das armadilhas colocadas na área, ou também a ocorrência de uma flutuação populacional desta espécie, onde segundo os mesmos autores a população destes insetos apresentaram um pico populacional no final da estação chuvosa, época em que a alta taxa de sobrevivência de pupa e casulos deste curculionídeo é atribuída à queda da ação de fungos e bactérias

No ambiente de savana, a umidade relativa afetou negativamente a abundância de $R$. palmarum, para o ambiente de floresta, o clima não influenciou a densidade destes besouros (Tabela 2).

Os resultados da análise de regressão múltipla tipo "stepwise" indicam que a umidade relativa do ar explicou o modelo de $23 \%$ e a temperatura média do ar de $16 \%$ no modelo ajustado para $R$. palmarum em ambiente de Savana (Tabela 3).

A combinação do feromônio de agregação com o atrativo alimentar (cana-de-açúcar) potencializou a eficiência de captura dos coleópteros nos plantios de Palma de Óleo nos ecossistemas de savana e floresta nesta pesquisa, dados que corroboram com FERREIRA et al. (2003), que afirma que a combinação da canade-açúcar com o feromônio de agregação da praga aumenta a atratividade de machos e de fêmeas de $R$. palmarum para as armadilhas, comprovando a ocorrência de sinergismo entre essas fontes atrativas.

Diante dos resultados, verificou-se que ocorreu flutuação de $R$. palmarum com picos populacionais nos meses de julho e agosto no ecossistema de savana e de abril a setembro no ambiente de floresta. A população de R. palmarum foi menor em meses de baixa precipitação pluviométrica nos dois ambientes avaliados com a Palma de Óleo em Roraima.

Tabela 2. Coeficientes de correlação de Pearson entre a abundância de $R$. palmarum capturados em armadilhas em plantio de palma-de-óleo em ambiente de Savana e Floresta em Roraima e dados de chuva (mm), umidade relativa - UR (\%) e a temperatura média do ar - TM ( $\left.{ }^{\circ} \mathrm{C}\right) 15$ dias antes da captura.

\begin{tabular}{ccccccc}
\hline \multirow{2}{*}{ Espécie } & \multicolumn{3}{c}{ Savana } & \multicolumn{3}{c}{ Floresta } \\
\cline { 2 - 7 } & Chuva & UR & TM & Chuva & UR & TM \\
\hline Rhynchophorus palmarum & $-0,27 \mathrm{~ns}$ & $-0,48^{*}$ & $-0,03 \mathrm{~ns}$ & $-0,07 \mathrm{~ns}$ & $0,02 \mathrm{~ns}$ & $-0,23 \mathrm{~ns}$ \\
\hline
\end{tabular}

* Correlação significativa pelo teste "t" de Student ( $p<0,05)$; ns - não significativo.

Tabela 3. Modelos ajustados pelo método "stepwise" da abundância de $R$. palmarum em plantio palma-de-óleo em ambiente de savana e os fatores meteorológicos (Chuva, Umidade Relativa - UR (\%) e Temperatura Média - TM $\left({ }^{\circ} \mathrm{C}\right)$. Boa Vista RR, 2011.

\begin{tabular}{ccccccc}
\hline Espécie & Ambiente & Variáveis & Coeficientes & $\mathbf{R}^{2(1)}$ (parcial) & $\mathbf{R}^{\mathbf{2}}$ (modelo) & Teste $\mathbf{F}^{(2)}$ \\
\hline & & Constante & 773,87 & - & 0,39 & $4,55^{* *}$ \\
\multirow{2}{*}{ Rhynchophorus palmarum } & Savana & UR & $-142,31$ & $0,23^{* *}$ & - & - \\
& & T.M. & $-445,82$ & $0,16^{*}$ & - & - \\
\hline
\end{tabular}

$\mathrm{R}^{2}$ : Coeficiente de determinação; (1) ${ }^{* *} ;{ }^{*}$ - significativo a $1 \%$ e $5 \%$ de probabilidade pelo teste "t" de Student; (2) ** - significativo a $1 \%$ de probabilidade.

\section{REFERÊNCIAS}

Alpizar, D., M. Fallas, A.C. Oehlschlager, L.M. Gonzalez, C.M. Chinchilla \& J. Bulgarelli, 2006. Pheromone mass trapping of the west indian sugarcane weevil and the american palm weevil (Coleoptera: Curculionidae) in palmito palm. Florida Entomologist, 85: 426-430.
Araújo, W.F., A.S. Andrade Júnior, R.D. Medeiros \& R.A. Sampaio, 2001. Precipitação pluviométrica mensal provável em Boa Vista, Estado de Roraima, Brasil. Revista Brasileira de Engenharia Agrícola e Ambiental, 5: 563-567.

Barbosa, R.I., 1997. Distribuição das chuvas em Roraima, p. 325-335. In: Barbosa, R.I. E. Ferreira \& E. Castellón, (Eds.), Homem, Ambiente e Ecologia no Estado de Roraima. INPA, 
Manaus. 613 p.

Bulgarelli-Mora, J., C. Chinchilla \& C. Oehlschlager, 1998. The red ring/little leaf syndrome and Metamasius hemipterus captures in oil palm in Costa Rica. ASD Oil. Palm Papers, 18: 17-24.

Cysne, A.Q., B.A Cruz, R.N.V. Cunha \& R.N.C. Rocha, 2013. Flutuação populacional de Rhynchophorus palmarum (L.) (Coleoptera: Curculionidae) em palmeiras oleíferas no Amazonas. Acta Amazonica, 43: 197-202.

Costa, E.C., M. D’Avila, E.G. Cantarelli, A.B. Murari \& C.G. Manzoni, 2014. Entomologia Florestal. Santa Maria, UFSM, $256 \mathrm{p}$.

Chinchilla, C.M, 1992. El síndrome del anillo rojo-hoja pequenã em palma aceitera y cocotero. Revista palma, 13: 33-56.

Chinchilla, C.M \& A.C. Oehlschlager, 1992. Captures of Rhynchophorus palmarum in traps baited with the maleproduced aggregation pheromone. ASD Oil Palm Papers, 5: 01-08.

Duarte, A.G., I.S. Lima, D.M.A.F. Navarro \& A.E.G. Sant'Ana, 2003. Captura de Rhynchophorus palmarum L. (Coleoptera: Curculionidae) em armadilhas iscadas com o feromônio de agregação e compostos voláteis de frutos do abacaxi. Revista Brasileira de Fruticultura, 25: 81-84.

Duarte, A.G., I.S. Lima, J.V. Araújo Júnior, A.G. Duarte, A.L.S. Albuquerque \& M.M. Cruz, 2008. Disposição do nematóide Bursaphelenchus cocophilus (Cobb) baujard, em coqueiros portadores da doença anel-vermelho. Revista Brasileira de Fruticultura, 30: 622-627.

Fachinello, J.C., C.S. Tibola, M. Vicenzi, E. Parisotto \& M.L.T. Matos, 2003. Produção integrada de pêssegos: três anos de experiência na região de Pelotas - RS. Revista Brasileira de Fruticultura, 25: 256-258.

Ferreira, J.M.S., M.L.S. Leal, F.B. Sarro, R.P.C. Araújo \& J.I.L. de Moura, 2003. Avaliação de diferentes fontes atrativas e suas prováveis interações na captura de Rhynchophorus palmarum. Manejo Integrado de plagas y Agroecologia, 67: 23-29.

Gallo, D., O. Nakano, S. Silveira Neto \& R.P.L. Carvalho, 2002. Manual de entomologia agrícola. Piracicaba, FEALQ, 920 p.

Krug, C., D.M.C. Bittencout, E. Barcelos, M.R.L. Rodrigues, P.C.S. Angelo, R.N.C. Rocha, R.N.V. Cunha, R.C. Quisen, R. Lopes, S.A. Rios \& W.A.A. Lima, 2013. Plano estratégico da Embrapa Amazônia Ocidental para a cultura do dendezeiro. Manaus: EMBRAPA (Documentos, 102), 73 p.

Morales, J.J. \& C.M.L. Chinchilla, 1991. Estudios poblacionales en Rhynchophorus palmarum L. y su relación com la enfermedad del Anillo rojo / Hoja pequeña em palma acitera en Costa Rica. Turrialba, 40: 475-478.

Moura, J.I.L., R. Toma, R.B. Sgrillo \& J.H.C. Delabie, 2006. Natural Efficiency of Parasitism by Billaea rhynchophorae (Blanchard) (Diptera: Tachinidae) for the control of Rhynchophorus palmarum (L) (Coleoptera: Curculionidae). Neotropical Entomology, 35: 273-274.
Navarro, D.M.A.F., M.M. Murta, A.G. Duarte, I.S. Lima, R.R. Nascimento \& A.E.G. Sant'Ana, 2002. Aspectos práticos relacionados ao uso do Rincoforol, o feromônio de agregação da Broca-do-olho-do-coqueiro Rhynchophorus palmarum L. (Coleoptera: Curculionidae) no controle de pragas do coqueiro. Análise de sua eficiência em campo. Química Nova, 25: 32-36.

Oehlschlager, A.C., C. Chinchilla, D. Castillo \& L. Goncalez, 2002. Control of Red Ring Disease by Mass Trapping of Rhynchophorus palmarum (Coleoptera: Curculionidae). Florida Entomologist, 85: 507-513.

Pinho, R.C., 2015. Distribuição Espacial de Rhynchophorus palmarum (Linnaeus, 1758) (Coleoptera: Curculionidae) em um Agroecossistema de Palma de Óleo na Amazônia Oriental. Dissertação (Mestrado em Agronomia) - Universidade Federal Rural da Amazônia. 54 p.

Santos, E.A., 2010. Caracterização de dendezeiros subespontâneos com base na produção de frutos e cachos. Dissertação (Mestrado em Produção Vegetal: Melhoramento de plantas e biotecnologia) - Universidade Estadual de Santa Cruz. 74 p.

Sanways, M.J., 1995. Invertebrate Conservation Research. Centre Department of Zoology and Entomology University of Natal. Pietermaritzburg, Chapman e Hall. 358 p.

Silveira Neto, S., R.C. Monteiro, R.A. Zucchi \& R.C.B. Moraes, 1995. Uso da análise faunística de insetos na avaliação do impacto ambiental. Scientia Agrícola, 52: 9-15.

Takada, H.M., A. Batista Filho, H. Hojo \& A.G. Carvalho, 2011. Flutuação populacional de Rhynchophorus palmarum no município de São Bento do Sapucaí, SP. Biológico, 73: 45-51.

Thomazini, M.J. \& A.P.B.W. Thomazini, 2000. A fragmentação florestal e a diversidade de insetos nas florestas tropicais úmidas. Rio Branco: EMBRAPA (Documentos, 57), 21 p.

Tiglia, E.A., E.F. Vilela, J.I.L. Moura \& N. Anjos, 1998. Eficiência de armadilhas com feromônio de agregação e cana-de-açúcar na captura de Rhynchophorus palmarum (L.). Anais da Sociedade Entomológica do Brasil, 27: 177-183.

Zorzenon, F.J., E.C. Bergmann \& J.E.A. Bicudo, 200o. Primeira ocorrência de Metamasius hemipterus e Metamasius ensirostris (Coleoptera: Curculionidae) em palmiteiros dos gêneros Euterpe e Bactris no Brasil. Anais do Instituto Biológico, 67: 265-268.

\section{Recebido em: 15/11/2014 \\ Aceito em: 12/04/2015}

\section{Como citar este artigo:}

Correia, R.G., A.C.S. Lima, A.C.C. Cordeiro, F.C. da S. Maciel, W.B.R. Martins \& L.F.S. Dionísio, 2015. Flutuação Populacional de Rhynchophorus palmarum L. (Coleoptera: Curculionidae) em Plantios de Palma de Óleo em Roraima. EntomoBrasilis, 8 (2): 130-134. Acessível em: $\underline{\text { doi:10.12741/ebrasilis.v8i2.490 }}$
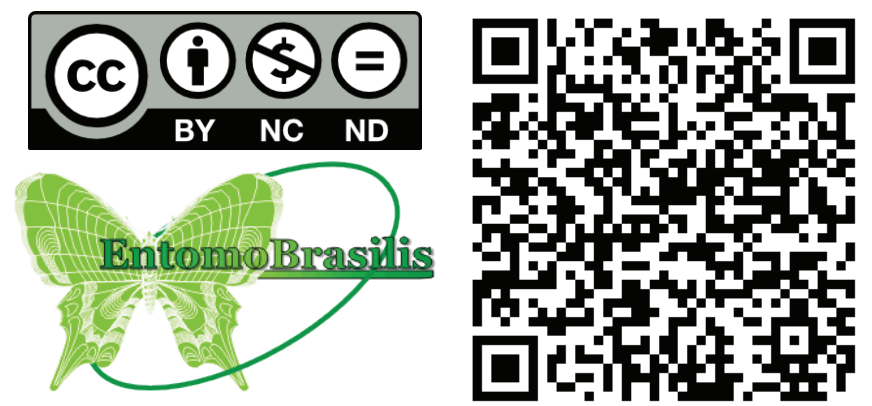